\title{
OFDM Systems Resource Allocation using Multi- Objective Particle Swarm Optimization
}

\author{
Rajendrasingh Annauth ${ }^{1}$ and Harry C.S.Rughooputh ${ }^{2}$ \\ ${ }^{1}$ Department of Electrical and Electronic Engineering, University of Mauritius, Reduit, \\ Mauritius \\ rannauth@mail.gov.mu \\ ${ }^{2}$ Department of Electrical and Electronic Engineering, University of Mauritius, Reduit, \\ Mauritius \\ r.rughooputh@uom.ac.mu
}

\begin{abstract}
Orthogonal Frequency Division Multiplexing (OFDM) has the inherent properties of being robust to interference and frequency selective fading and is de facto the adopted multiplexing techniques for the $4^{\text {th }}$ generation wireless network systems. In wireless system, resources such as bandwidth and power are limited, intelligent allocation of these resources to users are crucial for delivering the best possible quality of services. In this paper the problem of resource allocation in multiuser OFDM system is tackled using multi objective particle swarm optimization. Simulation results are presented for 3GPP-LTE system.
\end{abstract}

\section{KERWORDS}

Multi-Objective Particle Swarm Optimization, OFDM, Resource Allocation.

\section{INTRODUCTION}

OFDM is a combination of modulation and multiplexing. As modulation scheme, it is suitable to handle harsh and adverse environmental condition, whereas as multiple access scheme it offers high spectral efficiency and diversity. Applications include wireless multimedia and Internet access and it stands as a potential candidate for next generation wireless communication systems. Multiuser OFDM system has the inherent advantages of being flexible in subcarriers allocation, adaptive to channel condition, provision of high spectral efficiency, lower receiver complexity and simpler implementation by inverse fast Fourier transform (FFT) and FFT. In addition, OFDM has the merits of mitigating the effect of frequency selective fading and inter symbol interference, inherent in high data rate transmission, by converting frequency selective channel into a number of parallel narrowband flat fading sub-channels

A major problem in multiuser OFDM system is rate and power allocation to subcarriers. Two classes of resource allocation exist - the Fixed Resource Allocation (FRA) and Dynamic Resource Allocation (DRA). Assignment in FRA schemes is non optimal, since scheme is fixed regardless of channel conditions. On the other hand, DRA schemes are based on users channel gains and make full use of multiuser diversity to achieve higher performance. In dynamic multiuser literature, two classes of optimisation techniques have been proposed, specifically Margin Adaptive objective to minimize the overall transmit power under data constraint, and Rate Adaptive objective maximization of the data rate under power constraint. These optimization problems are nonlinear and computationally intensive to solve as complexity increases exponentially with constraints and variables [1]. The solution space of OFDM allocation problem is too large and conventional algorithms cannot solve it effectively.

DOI : $10.5121 /$ ijcnc.2012.4419 
Intelligent techniques such as genetic algorithms (GAs), simulated annealing, particle swarm optimization (PSO), Tabu search and others offer more speedy and robust solutions. Genetic algorithms have been widely applied for the OFDM resource allocation [2]. An alternate tool to GAs is PSO. PSO is a search optimization technique to find the best optimal solution through collaboration and information sharing among individuals in the swarm.

Resource allocation in multiuser OFDM for subcarriers, power and bits are conflicting objectives that require multi-objective approach for Pareto optimal non-dominated solution. Traditionally these are solved by combining all objectives into a single objective function. This solution is largely dependent on numerical weights or utility functions that are often difficult to select or selected arbitrarily. We are proposing the application of multi-objective PSO (MOPSO) for the resource allocation in multiuser OFDM considering rate power and subcarrier allocation constraints. In the formulation of the MOPSO, many ideas such as crowding distance, mutation operator and constraint handling are borrowed from the genetic evolutionary algorithm as suggested in the works of Raquel [3] and Deb [4].

\section{Related Works}

There have been a lot of studies on resource allocation in OFDM systems. In [5], Jang and Lee have proved that capacity is maximised when sub channel is assigned to user with the best sub channel gain and power is distributed by the water-filling algorithm. Rhee and Cioffi [6] studied the max-min problem, where by maximising the worst user's capacity, fairness in data rate is assured. Similarly, Shen et al. [1] proposed an optimal power allocation algorithm that maximizes the sum capacity, whereas Wong [7] has formulated OFDMA resource allocation problems that maximize the ergodic rates based dual optimization techniques. These classical approaches are inherently complex and NP hard. Resource allocation using Genetic Algorithm [8] has given better results than conventional algorithms for optimum power allocation. But only subcarrier allocation has been considered and bit loading was done using water-filling algorithm. In these works, the subcarrier, bit and power allocation problems have been formulated as a single objective optimization function. Annauth and Rughooputh [9] have efficiently applied the Deb's method of Non-dominated Sorting Genetic Algorithm (NSGA II) [10] to multiuser OFDM resource allocation with improved performance and faster rate of convergence.

In PSO, each particle flies in the search space with a velocity which is dynamically adjusted according to its own flying experience and its companions' flying experiences. It is widely reported that PSO algorithm is very easy to implement to solve real world optimization problems [11] and has fewer parameters to adjust when compared to other evolutionary algorithms. The information sharing mechanism among the particles in PSO is significantly different from the information sharing among the chromosomes in GAs. In GAs, the entire group moves towards an optimal solution area. However, in PSO only the global best or local best solution is reported to other particles in a swarm. Therefore, evolution only looks for the best solution and the swarm tends to converge to the best solution quickly and efficiently.

Gheitanchi et al. [12] has applied PSO for subcarrier allocation in OFDMA systems with significant reduction of computational complexity and increase flexibility compared to conventional techniques, whereas, Chakravarthy et al. PSO resource allocation techniques [13] has improved delay characteristics while maintaining fairness and throughput utilization. Shu' aibu's approach [14] uses $22.5 \%$ less CPU time than other techniques. Yang Yi et al. [15] has used a combination of GA and PSO in the subcarrier and power allocation with improved performance than normal PSO. Ahmed [16] has shown that the Differential Evolution techniques is better than PSO but takes more time to converge. In literature, the use of PSO for OFDM resource allocation have so far been based on single objective optimization technique, we are therefore investigating its extension to handle multi-objective problems. 
International Journal of Computer Networks \& Communications (IJCNC) Vol.4, No.4, July 2012

\section{PROBLEM FORMULATION}

Consider a multiuser OFDM system where different $K$ users are allocated to the $N$ subcarriers, and each subcarrier $n$ is assigned power $P_{k, n}$. Each of the user's bits are then modulated into $N$ $M$-level QAM symbols, which are subsequently combined using the inverse fast Fourier transform into an OFDM symbol. This is then transmitted through a slowly time-varying frequency-selective Rayleigh channel with bandwidth $B$. The subcarrier allocation is made known to all the users through a control channel; hence each user needs only to decode the bits on their assigned subcarriers.

It is assumed that each user experiences independent fading and the channel gain of user $k$ in subcarrier $n$ is denoted as $g_{k, n}$, with additive white gaussian noise (AWGN) $\mathrm{s}^{2}=N_{0} \frac{B}{N}$ where $N_{0}$ is the noise power spectral density. The corresponding subchannel signal-to-noise ratio (SNR) is thus denoted as $h_{k, n}=\frac{g_{k, n}^{2}}{S^{2}}$ and the $K^{\mathrm{th}}$ user's received SNR on subcarrier $\mathrm{h}$ is $\mathrm{g}_{\mathrm{k}, \mathrm{n}}=p_{k, n} h_{k, n}$. The slowly time-varying assumption is crucial since it is also assumed that each user is able to estimate the channel perfectly and these estimates are made known to the transmitter via a dedicated feedback channel. These channel estimates are then used as input to the resource allocation algorithms.

\subsection{OBJECTIVES FUNCTIONS}

In order that the bit error rate (BER) constraints be met, the effective SNR has to be adjusted accordingly. The BER of $M$-level QAM as a function of received SNR $\mathrm{g}_{\mathrm{k}, \mathrm{n}}$ and number of bits $r_{k, n}$ can be approximated to within $1 \mathrm{~dB}$ for $r_{k, n} \geq 4$ and $\mathrm{BER} \leq 10^{-3}$ as [17]

$$
B E R_{M Q A M}\left(\mathrm{~g}_{\mathrm{k}, \mathrm{n}}\right) \sim 0.2 \exp \left[\frac{-1.6}{2^{r} k, n-1}\right]
$$

Solving for $r_{k, n}$

$$
r_{k, n}=\log _{2}\left(1+p_{k, n} H_{k, n}\right)
$$

The objective function of the resource allocation is formulated as

$$
\max _{c_{k, n}: p_{k, n}} \frac{B}{N} \sum_{n}^{k} \sum_{n=1}^{N} C_{k, n} \log _{2}\left(1+p_{k, n} H_{k, n}\right)
$$

\subsection{MULTI- OBJECTIVE FORMULATION}

We aim to maximize the data rate among all the users subject to the constraint that the total power cannot exceed a given value. Therefore, we modify the power constraint slightly and assume that the total available transmission power is limited to a certain range with a typical value $P_{T}$. So, we have a second objective to bring the total power as close to $P_{T}$ as possible. The new multi-objective optimization problem is

$$
p_{k, n, 1}^{\max } 1_{k, n}^{K} \sum_{K=1}^{K} R_{k}
$$


International Journal of Computer Networks \& Communications (IJCNC) Vol.4, No.4, July 2012

$$
\begin{gathered}
\text { Maximize } R_{k}=\sum_{n=1}^{N} \frac{1_{k, n}}{N} \log _{2}\left(1+\frac{P_{k, n} h_{k, n}^{2}}{N_{0} \frac{B}{N}}\right) \\
\text { Minimize } \sum_{K=1}^{K} \sum_{n=1}^{N} P_{k, n} \leq P_{\text {total }}
\end{gathered}
$$

subject to the constraints detailed below

$$
\begin{array}{ll}
\text { C1: } & C_{k, n} \in\{0,1\} \forall \kappa, n \\
\text { C2: } & \sum_{k=1}^{K} c_{k, n}=1, \forall_{n} \\
\text { C3: } & p_{k, n} \geq 0 \forall \kappa, n \\
\text { C4: } & \sum_{k=1}^{K} \sum_{n=1}^{N} c_{k, n} p_{k, n} \leq P_{\text {total }}
\end{array}
$$

Constraints $\mathrm{C} 1$ and $\mathrm{C} 2$ are on the subcarrier allocation to ensure that each sub channel is assigned to one user. Constraints $\mathrm{C} 3$ and $\mathrm{C} 4$ are on the power allocation where $P_{\text {total }}$ is the total transmit power of the system.

\subsection{MULTI-OBJECTIVE PARTICLE SWARM OPTIMIZATION}

PSO is a population- based stochastic optimization technique developed by Eberhart and Kennedy [18] inspired by the social behaviour of flocks of bird. The PSO is initialized with a population of random solutions and this initial population evolves over generation to find optima. In PSO each particle in the population has a velocity, which enables it to fly through the problem space. Therefore, each particle is represented by a position and velocity vector. Dimensions of position and velocity vectors are defined by the number of decision variables in optimization problem. Modification of the position of a particle is performed by using its previous position information and its current velocity. Let $\bar{x}_{i}(t)$ denote the position of particle $p_{i}$, at time step $t$. The position of $p_{i}$ is then changed by adding a velocity $\bar{v}_{i}(t)$ to the current position, i.e.:

$$
\left.\bar{x}_{i}(t)=\bar{x}_{i}(t-1)+\bar{v}_{i}(t)\right)
$$

According to the value of the objective function, each particle knows its best position ever (personal best - pbest) and (global best -gbest) among all personal bests. For a single objective problem, the result of optimization problem will be the position vector of gbest at final iteration. These principles can be formulated as

$$
\bar{v}_{i}(t)=\bar{v}_{i}(t-1)+C_{1} r_{1}\left(\bar{x}_{\text {pbest }}-\bar{x}_{i}(t)\right)+
$$


where $C_{1}$ and $C_{2}$ are the cognitive and social learning factors (usually defined as constant), and $r_{1}, r_{2} \in[0,1]$ are random values. $C_{1}$ and $C_{2}$ constant are normally assigned value 2.0 for all applications.

The version of PSO given above is not suitable for solving multi-objective optimization problems since there is no absolute global minimum. Therefore, the algorithm needs some modification to locate the Pareto front in multi-objective optimization problems. With multiobjective optimization we aim to find a set of different solutions (the Pareto Optimal set) with a single run and this is achieved by maximizing the number of elements of Pareto optimal set, minimizing the distance of the Pareto front produced by our algorithm with respect to the true (global) Pareto front and maximizing the spread of solutions found, so that we can have a distribution of vectors as smooth and uniform as possible. The following issues are also considered when extending PSO to multi-objective optimization

- Selection of particles (to be used as leaders) in order to give preference to non dominated solutions over those that are dominated.

- Retention of non dominated solutions found during the search process in order to report solutions that are non dominated with respect to all the past populations and not only with respect to the current one. It is desirable that these solutions are well spread along the Pareto front.

- Maintenance of diversity in the swarm in order to avoid convergence to single solution.

When solving single objective optimisation problems, the leader that each particle uses to update its position is completely determined once a neighbourhood topology is established. However, in the case of multi-objective optimization problems, each particle might have a set of different leaders from which just one can be selected in order to update its position. Coello et al [19] have proposed the idea of having an external archive in which every particle will deposit its flight experiences after each flight cycle. The updates to the external archive are performed considering a geographically-based system defined in terms of the objective function values of each particle. The search space explored is divided on hypercube. Each hypercube receives a fitness value based on the number of particles it contains. Thus, in order to select a leader from each particle of the swarm, a roulette wheel selection using these fitness values is first applied, to select the hypercube from which the leader will be taken. Once the hypercube has been selected, the leader is randomly chosen. This approach also uses a mutation operator that acts both on the particles of the swarm, and on the range of each design variable of the problem to be solved.

We have incorporated the mechanism of crowding distance computation into the algorithm specifically on global best selection and in deletion method of external archive of non dominated solutions as suggested in the works of C.R. Raquel et al. [3] and Deb [4]. The crowding distance mechanism together with the mutation operator maintains the diversity of non dominated solutions in the external archive.

The pseudo code of the MOPSO algorithm is given in Figure 1. 


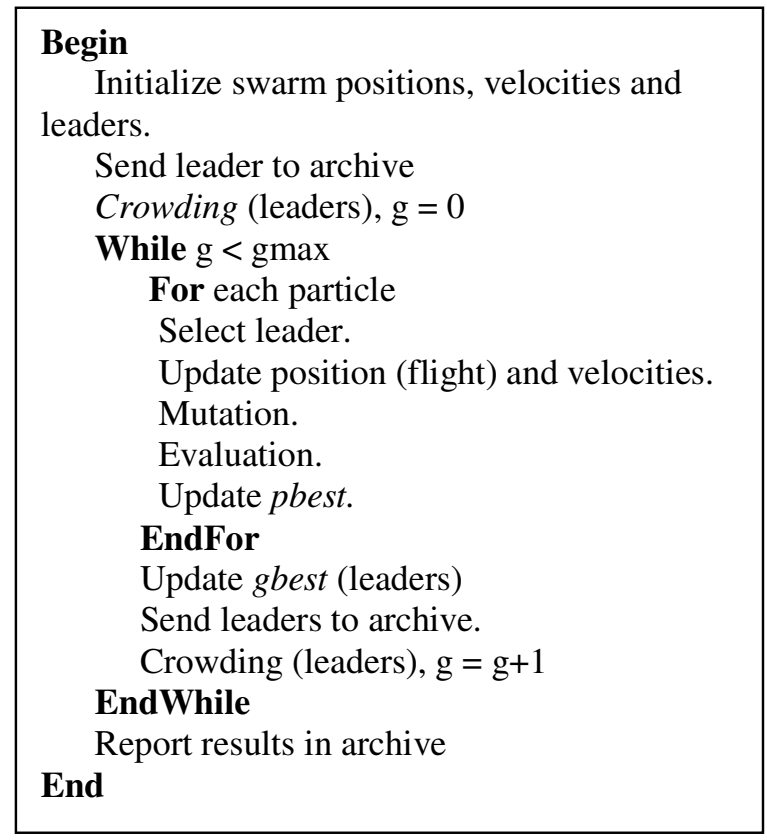

Figure 1. Pseudo code for MOPSO Algorithm.

\subsection{RESOURCE ALLOCATION USING MOPSO}

We consider a multi-user OFDM system with $N$ subchannels and $K$ users with total transmitted power of P_total. Our objective is to optimize the subcarriers and power allocation in order to achieve the highest capacity under the total power constraint. For the joint allocation of bits and power, the swarm particles position and velocity have to be defined. Therefore, a channel matrix $H$ of $K$ rows and $N$ columns, each of the elements denoting the channel gain is generated.

$$
H=\left[\begin{array}{ccc}
p_{1,1} & \cdots & p_{1, N} \\
\vdots & \ddots & \vdots \\
P_{k, 1} & \cdots & P_{k, N}
\end{array}\right]
$$

From matrix $H$, velocity is initially set to zero and the initial value of the channel gain assigned to the particle position. Bits are allocated to the subcarriers corresponding to the respective channel gain, and the fitness value for the capacity and allocated power are computed in accordance to equation 5 and 6 .

The positions of the particles that represent non-dominated vectors are stored in the external archive A. The crowding distance values of each non dominated solution in archive A are calculated and the non-dominated vectors are sorted in descending orders to select a set of leaders. At each generation, for each particle, a leader is selected and the flight is performed. The velocity and position of each particle are updated as follows

$$
\begin{gathered}
V[i]=W \times V[i]+R_{1} \times(P B E S T S[i]-P[i]+ \\
\left.\left.R_{2} \times A[G B E S T]-P[i]\right)\right) \\
\bar{x}_{i}(t)=\bar{x}_{i}(t-1)+\bar{v}_{i}(t)
\end{gathered}
$$

The PBEST and GBEST are thus determined. The nondominated vectors are stored in archive A. The crowding distance of each nondominated particle is determined. Mutation operation is 
then applied so as to promote diversity within the population. Then the particle is evaluated and its corresponding PBEST is updated. A new particle replaces the PBEST particle usually when this particle is dominated or if both are incomparable (i.e., they are both non-dominated with respect to each other). After all the particles are updated, the set of leaders are updated, too.

The steps of the MOPSO algorithm is iteratively repeated until the termination criterion is met such as maximum number of generations or when there has been no change in the set of nondominated solutions found for a given number of generations. The output of the MOPSO method is the set of non-dominated solutions stored in the final archive.

\subsection{SIMULATION}

The MOPSO algorithm has been used to allocate subcarriers and power to the multiuser OFDM system. The MOPSO parameters used are given in TABLE I for OFDM using 128 subcarriers.

TABLE I: Simulation parameters

\begin{tabular}{|c|c|}
\hline$V[i]$ & Old velocity of particle $i$ along dimension $d_{i}$ \\
\hline$V[i]$ & New velocity of particle $i$ along dimension $d_{i}$ \\
\hline$W$ & Inertia weight which is usually between 0.8 to 1.2 \\
\hline $\bar{x}_{i}(t)$ & Current position of particle $i$ along dimension $d_{i}$ \\
\hline PBESTS[i] & The Personal best solution of particle i along \\
\hline$\left[\begin{array}{c}\text { GBEST }] \\
\text { dimension } d_{i}\end{array}\right.$ & $\begin{array}{c}\text { The global best solution the whole population ever } \\
\text { along dimension } d_{i}\end{array}$ \\
\hline$R_{1} R_{2}$ & Random numbers between 0 and 1 \\
\hline
\end{tabular}

The following experiments have been conducted to analyse the performance of the MOPSO

(a) The MOPSO versus MOPSO with the crowding distance (MOPSO-CD).

(b) The MOPSO-CD with mutation versus MOPSO-CD without mutation.

In the experiments conducted with the MOPSO, repository size of up to 25 particles and the mutation rates of 0.5 have been used. To restrict the random effects, the experiments were repeated thirty times. Each experiment uses a different randomly generated initial population. The non dominated solutions obtained for the bi-objective of subcarriers and power is shown in Figure 2. From Figure 2 and 3 it can be seen that both the MOPSO and the NSGA II algorithms [9] cover the entire Pareto front. However, for the MOPSO, there is discontinuity along the curve as the algorithm performs statistically better in a particular region. With the computation of the crowding distance, there is significant improvement in the distribution and diversity along the Pareto front of the algorithm as shown in Figure 4 for MOPSO CD. As can be 
observed from the graph, out of the 25 users, depending on the channel conditions, allocation of subcarriers and power have been provided to 20 users only. Mathematically, every Pareto optimal point is an equally acceptable solution. Using the procedures proposed by Miettinen [20], the best compromise solution is obtained through application of fuzzy set theory to each objective function. The best non-dominated solution can be found when equation is a maximum where the normalized sum of membership function values for all objectives is highest

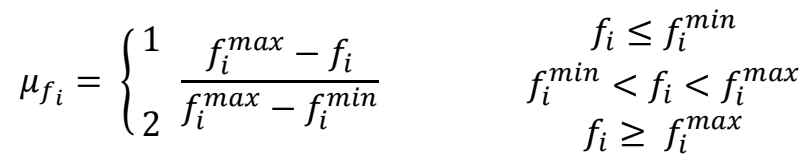

$$
\begin{aligned}
& \mu^{k}=\frac{\sum_{i=1}^{N} \mu_{f_{i}}^{k}}{\sum_{k=1}^{M} \sum_{i=1}^{N} \mu_{f_{i}}^{k}}
\end{aligned}
$$

where $M$ is the number of non-dominated solution and $N$ is the number of objective functions.

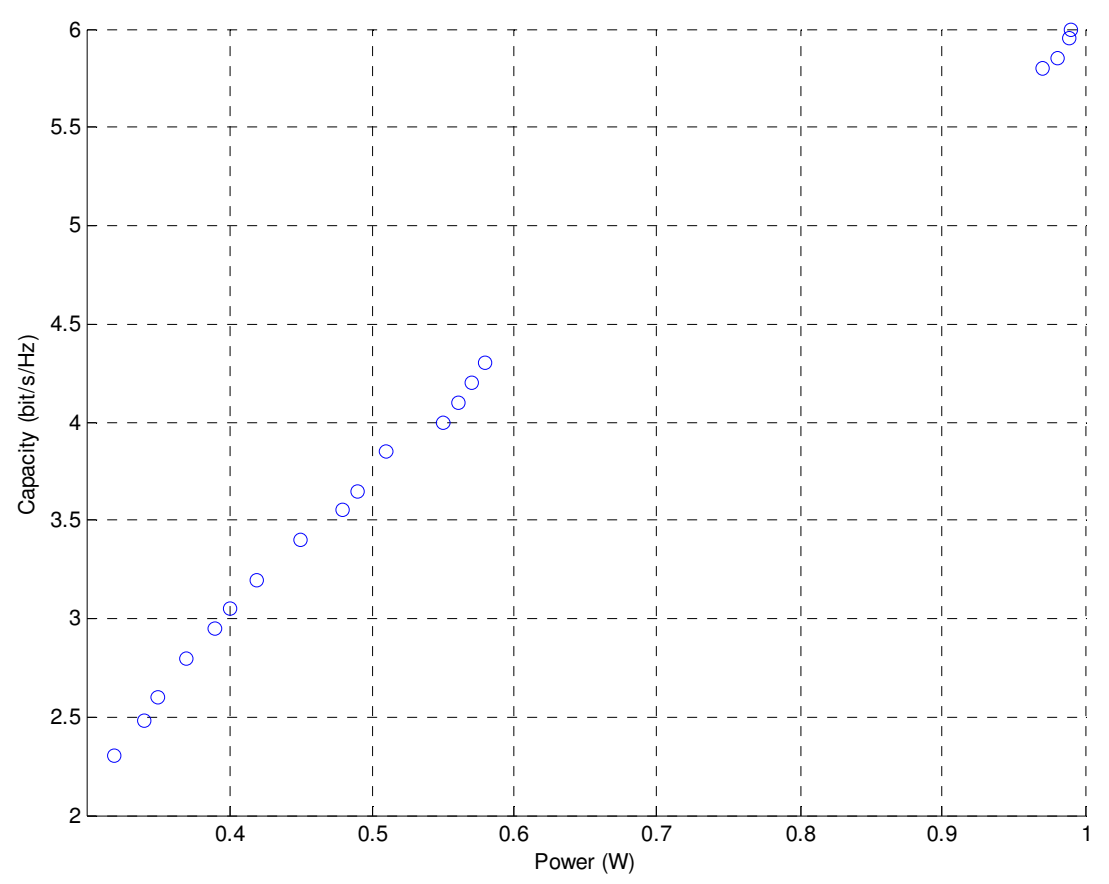

Figure 2. Optimal Pareto front for MOPSO Algorithm 
International Journal of Computer Networks \& Communications (IJCNC) Vol.4, No.4, July 2012

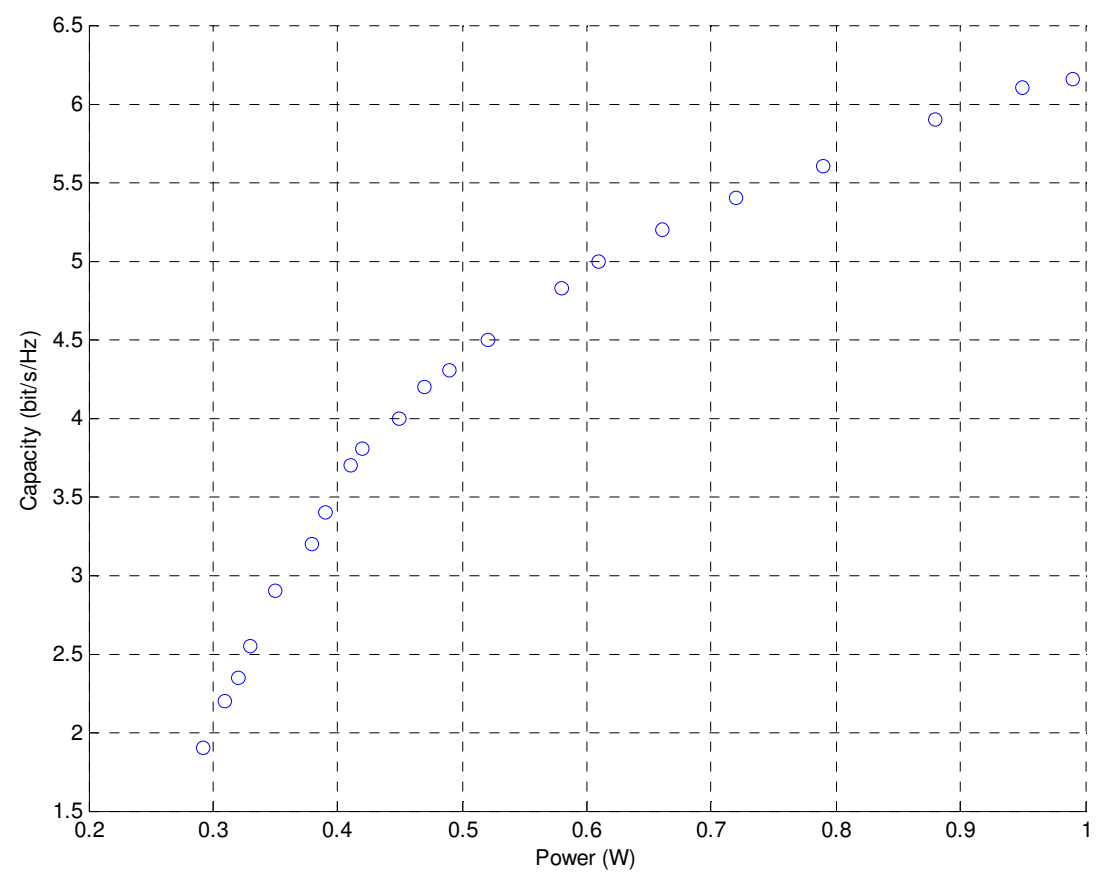

Figure 3. Pareto Optimal Front for NSGA II Algorithm.

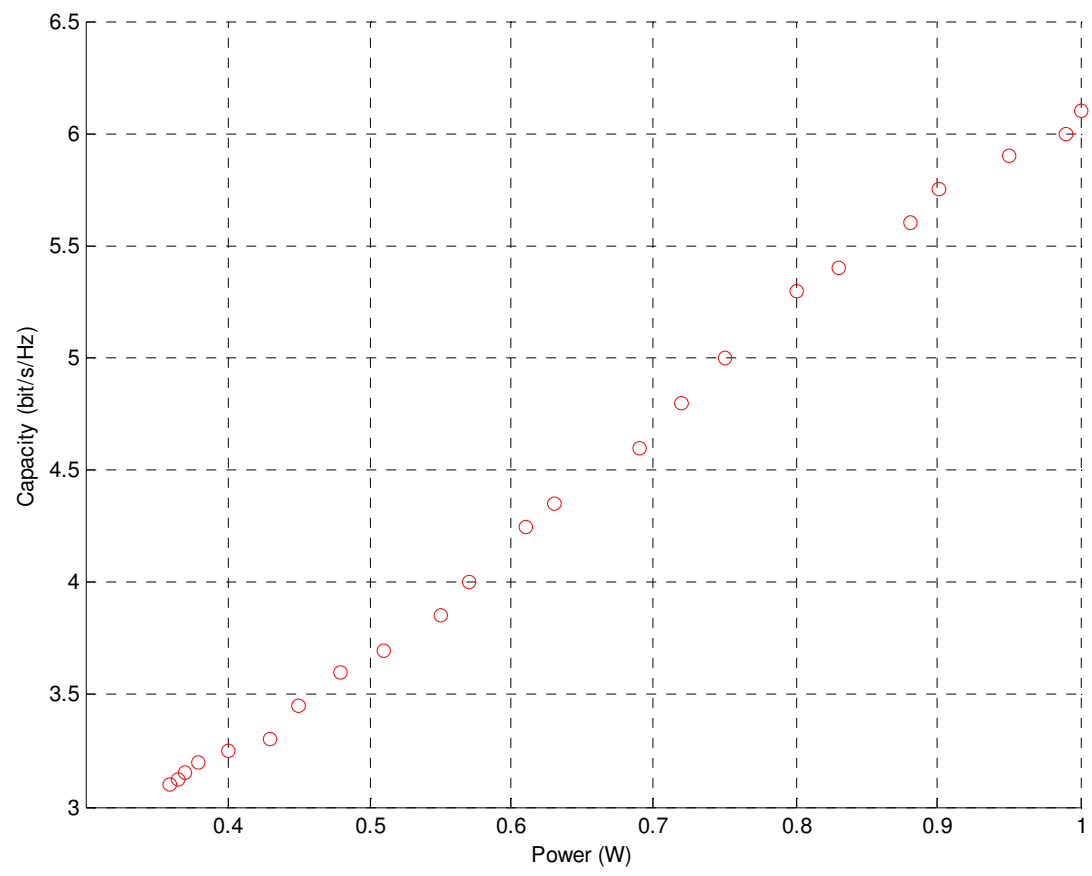

Figure 4. Optimal Pareto front for MOPSO CD Algorithm 
International Journal of Computer Networks \& Communications (IJCNC) Vol.4, No.4, July 2012

\subsection{PERFORMANCE METRICS}

As MOPSO is a novel method, it is necessary to compare it in terms of performance. Multiobjective optimization has two distinct goals:

(i) discover solution as close to the Pareto-optimal solutions as possible and

(ii) find solutions as diverse as possible in the obtained non-dominated front.

While comparing different evolutionary algorithms, Deb et al.[21] suggested that at least two performance metrics (one evaluating the progress towards the Pareto - Optimal front and the other evaluating the spread solutions) need to be used. The two performance metrics used for this study are

(a) Generational Distance as the Convergence Metric

Given a non-dominated set $Q$ and a Pareto-optimal set $P^{*}$, the Generational Distance (GD) metric calculates the average distance of the solutions of $Q$ and $P^{*}$, as follows (Van Veldhuzein [22]):

$$
G D=\frac{\left(\sum_{i=1}^{|Q|} d_{i}^{p}\right)^{1 / p}}{|Q|}
$$

For $P=2$, the parameter $d_{i}$ is the Euclidean distance (in the objective space) between the solution in $\mathrm{Q}$ and the nearest member $P^{*}$ :

$$
d_{i}=\min _{k=i}^{\left|P^{*}\right|} \sqrt{\sum\left(f_{m}^{(i)}-f_{m}^{*(k)}\right)^{2}}
$$

where $f_{m}^{*(k)}$ is the $m$-th objective function value of the $k$-th member of $p^{*}$.

An algorithm having a small value of GD is better. If the objective function values are of differing magnitude, they should be normalised before calculating the distance measure. A large number of solutions in $p^{*}$ is recommended in order to a make distance calculation reliable.

(b) Spread as Diversity Metric

Additionally, Deb et al.[21] has proposed the spread metric for evaluating diversity among non-dominated solutions

$$
\Delta=\frac{\sum_{m=1}^{M} d_{m}^{e}+\sum_{i=1}^{Q}\left|d_{i}-\bar{d}\right|}{\sum_{m=1}^{M} d_{m}^{e}+|Q| \bar{d}}
$$

Where $d_{i}$ can be any distance measure between neighbouring solutions and $\bar{d}$ is the mean value of these distances measures. In this work $d_{i}$ is calculated using the Euclidean distance i.e. the sum of absolute differences in objective values or the crowding distance as defined at. $d_{m}^{e}$ is the distance between the extreme solutions of Q and $p^{*}$ for each objective. For two objectives problem, the term $|Q|$ is replaced by $|Q-1|$. For an ideal distribution of solutions, $\Delta=0$ and for bad distributions, $\Delta$ value can be more than one. Thus, an algorithm finding a smaller $\Delta$ value is able to find a better diverse set of non-dominated solutions. 
Besides these two performance metrics, Deb also suggested a convergence metric combining the GD and spread in order to evaluate both goals of convergence and diversity.

$$
\begin{gathered}
W=W_{1} G D+W_{2} \Delta \\
W_{1}+W_{2}=1
\end{gathered}
$$

An algorithm having a small value of $\mathrm{W}$ means that algorithm is good in both convergence and diversity preserving ability.

\subsection{RESULTS AND DISCUSSION}

To have an insight indication of the performance of the proposed algorithm, statistical analysis has been performed on the MOPSO, MOPSO CD and NSGA II. Tables II to V show the numerical results obtained in terms of the space and diversity performance metrics considered and their computational time.

TABLE II. Results of Convergence metric

\begin{tabular}{|c|c|c|c|c|}
\hline \multirow{2}{*}{ Metrics } & \multicolumn{4}{|c|}{ Generation Distance } \\
\hline & MOPSO & MOPSO CD & $\begin{array}{c}\text { Mutated } \\
\text { MOPSO CD }\end{array}$ & NSGA II \\
\hline Min & 0.1630 & 0.1445 & 0.1700 & 0.1670 \\
\hline Max & 0.2310 & 0.1900 & 0.2351 & 0.1859 \\
\hline Average & 0.2100 & 0.1645 & 0.2077 & 0.1764 \\
\hline Median & 0.2165 & 0.1600 & 0.2124 & 0.1760 \\
\hline $\begin{array}{c}\text { Standard } \\
\text { Deviation }\end{array}$ & 0.0228 & 0.0153 & 0.0195 & 0.0068 \\
\hline
\end{tabular}

TABLE III. Results of Diversity Metric

\begin{tabular}{|c|c|c|c|c|}
\hline \multirow{2}{*}{ Metrics } & \multicolumn{4}{|c|}{ Spread $\Delta$} \\
\hline & MOPSO & MOPSO CD & \multicolumn{1}{|c|}{ Mutated } & \\
& & & & \\
\hline Min & 0.1800 & 0.0800 & 0.0900 & 0.0550 \\
\hline Max & 0.4000 & 0.1900 & 0.1650 & 0.1690 \\
\hline Average & 0.2940 & 0.1192 & 0.1199 & 0.0992 \\
\hline Median & 0.2900 & 0.1138 & 0.1170 & 0.0950 \\
\hline $\begin{array}{c}\text { Standard } \\
\text { Deviation }\end{array}$ & 0.0621 & 0.0301 & 0.0229 & 0.0384 \\
\hline
\end{tabular}


TABLE IV. Results of Weighted Metric

\begin{tabular}{|c|c|c|c|c|}
\hline \multirow{2}{*}{ Metrics } & \multicolumn{4}{|c|}{ Weighted Metric $W$} \\
\hline & MOPSO & MOPSO CD & $\begin{array}{c}\text { Mutated } \\
\text { MOPSO CD }\end{array}$ & NSGA II \\
\hline Min & 0.1953 & 0.1010 & 0.1140 & 0.0895 \\
\hline Max & 0.3590 & 0.1780 & 0.7970 & 0.1729 \\
\hline Average & 0.2716 & 0.1330 & 0.1456 & 0.1224 \\
\hline Median & 0.2673 & 0.1290 & 0.1451 & 0.1179 \\
\hline $\begin{array}{c}\text { Standard } \\
\text { Deviation }\end{array}$ & 0.0441 & 0.0210 & 0.0189 & 0.0248 \\
\hline
\end{tabular}

TABLE V. Results of the computation time (in seconds)

\begin{tabular}{|c|c|c|c|c|}
\hline \multirow{2}{*}{ Metrics } & \multicolumn{4}{|c|}{ Time (s) } \\
\hline & MOPSO & MOPSO CD & $\begin{array}{c}\text { Mutated } \\
\text { MOPSO CD }\end{array}$ & NSGA II \\
\hline Min & 0.009 & 0.0550 & 0.0530 & 0.1437 \\
\hline Max & 0.023 & 0.0810 & 0.0842 & 0.3069 \\
\hline Average & 0.014 & 0.0652 & 0.0655 & 0.2255 \\
\hline Median & 0.012 & 0.0655 & 0.0655 & 0.2329 \\
\hline $\begin{array}{c}\text { Standard } \\
\text { Deviation }\end{array}$ & 0.0005 & 0.0085 & 0.0096 & 0.0546 \\
\hline
\end{tabular}

In term of solution diversity MOPSO-CD has a better distribution of the generated nondominated solutions than MOPSO. The performance of MOPSO-CD is almost twice better than that of MOPSO. This shows that the crowding distance helps in providing both a well distributed set of non-dominated solutions and helps in the converge of the algorithm in the true Pareto front. The MOPSO is five time faster than the MOPSO-CD. This is attributed to the adaptive grid used by the MOPSO which can be computed faster than crowding distance where the relative distances of each solution in the archive is computed whenever the global best solution is selected and when selecting a solution to be replaced by new solutions. Despite this the proposed MOPSO-CD is still competitive because aside from being able to converge to Pareto front, it also produces a well distributed set of the non-dominated solutions. In fact, this is more significant, when we compare the impact of the mutation operator on the MOPSO-CD. The results are shown in Tables II to V. The results also show that running MOPSO-CD with and without mutation computation are almost comparable.. In terms of diversity, there is a marginal difference in their performance. This is also true with regard to the computational time. The use of mutation operator is recommended to enhance and maintain diversity. 
It is of high importance to calculate the convergence time of evolutionary algorithms for practical implementation. First we see that MPSO shows better efficiency in execution time as the rate of convergence is higher compared to the other algorithms. Although it is evident from results that NSGA II outperforms MOPSO, yet their time requirement are relatively higher than MOPSO. It should be noted that in MOPSO, we count the number of position and velocity update used for the total generation, and for NSGA II, we count the number of equations for selection, cross over and mutation operations for total generations. MOPSO require less number of functions than NSGA II to reach a specified target value. So in terms of usage of functions, MOPSO is more efficient than NSGA II algorithm, although the later performs relatively better than MOPSO.

We consider an OFDM system based on a standard 3 GPP-LTE down link [23] with 128 subcarriers, 72 used subcarriers, $1.4 \mathrm{MHz}$ bandwidth. We have simulated the multiuser LTE down link transmission over the frequency selective fading channel using 1000 sub frames transmitted in an SNR range of 0 to $20 \mathrm{~dB}$ using adaptive modulation and bit allocation of the MOPSO algorithm. Figure 5 and 6 show the throughput and BER results of the evolutionary algorithms on a 3 GPP LTE platform [24]. An improvement of about 2 dB SNR gap is achieved for NSGA II and MOPSO CD without retransmission. This confirms and validates the MOPSO $\mathrm{CD}$ algorithm as an effective and viable method for resource allocation in the multiuser OFDM systems.

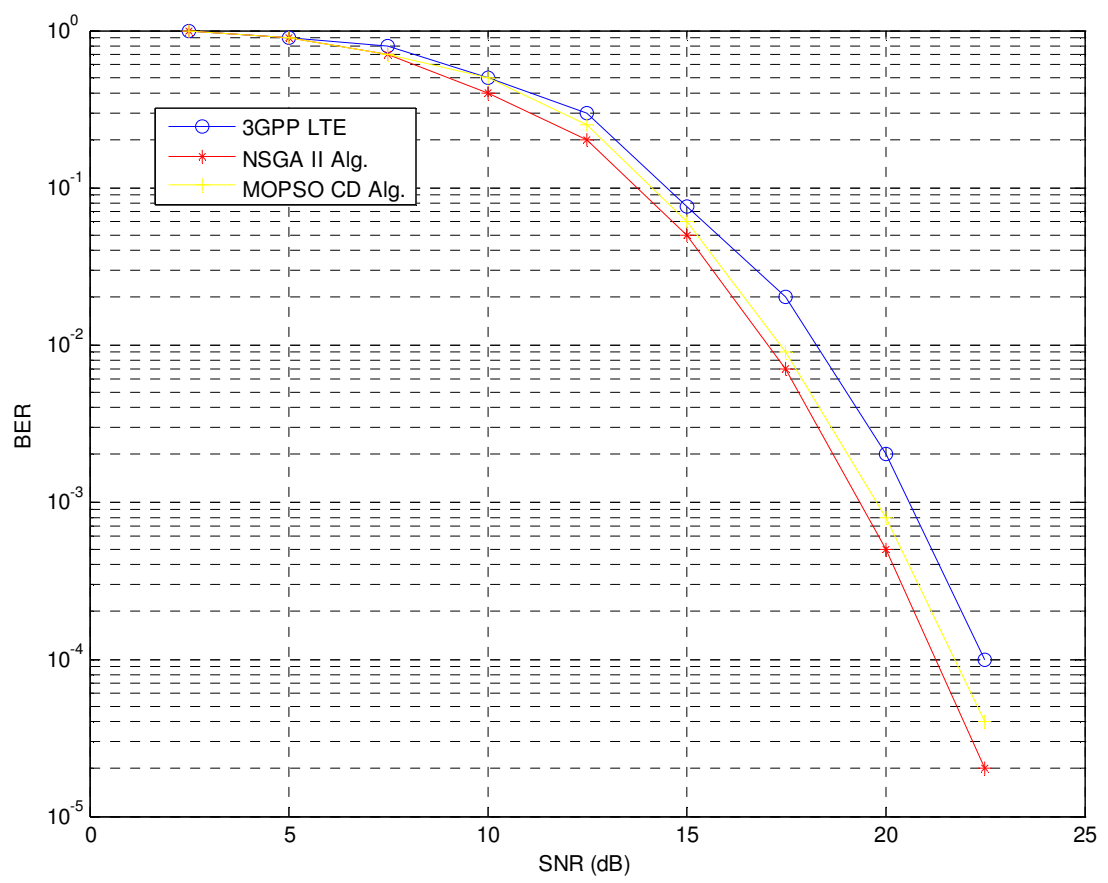

Figure 5. BER results for LTE, NSGA II and MOPSO CD 


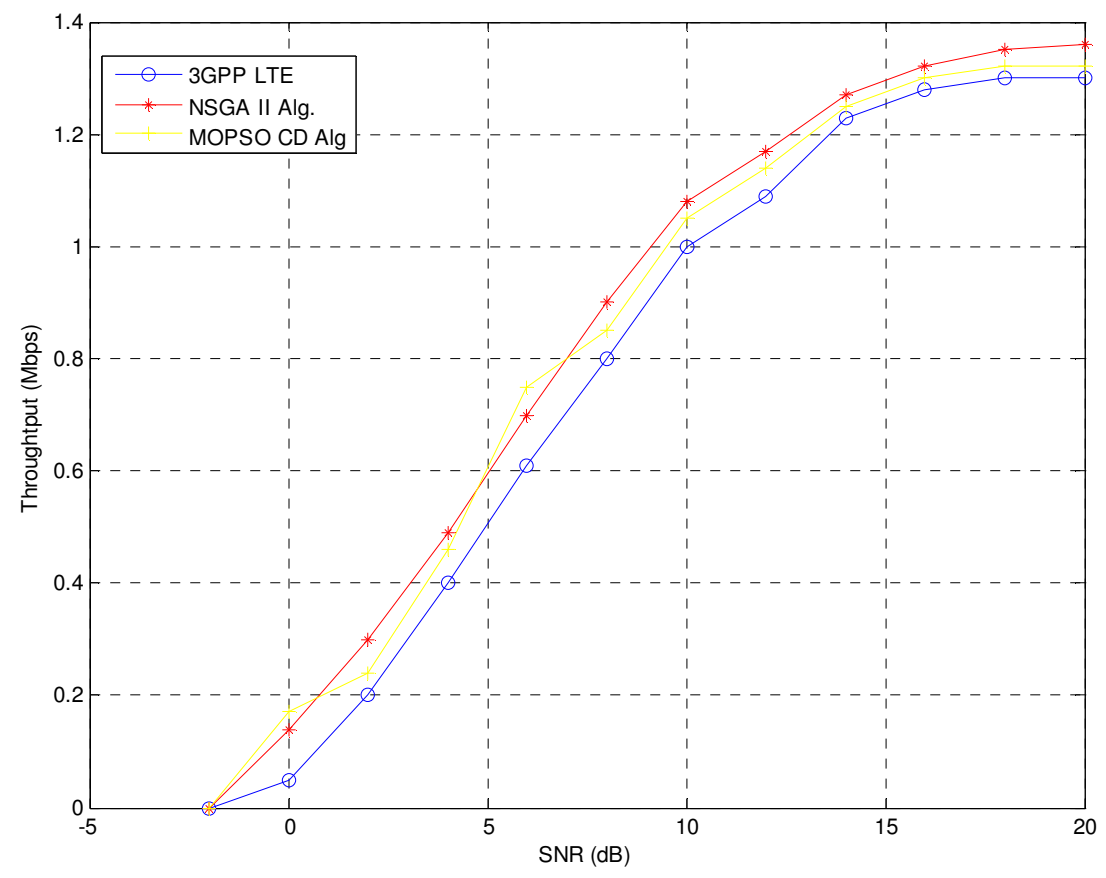

Figure 6. Throughput results for LTE, NSGA II and MOPSO CD

\subsection{CONCLUSIONS}

We have presented a proposal to extend PSO to handle multi-objective problems of resource allocation to multiuser OFDM system. The proposed algorithm is easy to implement and the exploratory capabilities of the PSO have been improved by introducing the mutation as well as crowding distance computation. The simulation results indicate that our approach is a viable alternative since it has average performance comparable to NSGA II, the fastest and elitist evolutionary algorithm. Additionally, the exceptionally low computational times required by the MOPSO make it a very promising approach to the resource allocation of the multiuser OFDM system, in which computational cost is a vital issue.

\section{ACKNOWLEDGEMENT}

I would like to thank the Tertiary Education Commission of Mauritius for the bursary awarded to carry out this research work at the University of Mauritius.

\section{REFERENCES}

[1] Zukang Shen, Jeffrey G. Andrews and Brian L. vans, "Optimal Power Allocation in Multiuser OFDM Systems”, Proc. IEEE Global Commun. Conf., San Francisco, CA, Dec. 2003, pp. 337341

[2] Y. B. Reddy, "Genetic Algorithm Approach for Adaptive Subcarrier, Bit, and Power Allocation" Proceedings of the 2007 IEEE International Conference in Networking, Sensing and Control, London, UK, 15-17 April 2007

[3] C.Raquel and P.C. Naval Jr., An effective use of Crowding Distance in Multi-objective Particle Swarm Optimization, GECCO 05, June 25 - 29,2005 
International Journal of Computer Networks \& Communications (IJCNC) Vol.4, No.4, July 2012

[4] K.Deb and N.Padhye, Improving a Particle Optimization Algorithm Using Evolutionary Algorithn Framework, KanGAL Report 2010003, February 2010.

[5] J.Jang and K.B.Lee, Transmit Power adaptation for Multiuser OFDM systems, IEEE Comunications Magazine, Vol 21, No.2, pp. 171 - 178, Feb 2003.

[6] W. Rhee and J.M. Cioffi," Increase in capacity of multiuser OFDM system using dynamic subchannel allocation," in Proc. IEEE Vehicular Technology Conf. (VTC'2000), 2000, PP. 10851089.

[7] I. C. Wong and B. L. Evans, "Optimal Downlink OFDMA Resource Allocation with Linear Complexity to Maximize Ergodic Rates", IEEE Transactions on Wireless Communications, vol. 7, no. 3, Mar. 2008, pp. 962-971.

[8] Y. B. Reddy, "Genetic Algorithm Approach for Adaptive Subcarrier, Bit, and Power Allocation" Proceedings of the 2007 IEEE International Conference in Networking, Sensing and Control, London, UK, 15-17 April 2007

[9] R. Annauth and H.C.S.Rughooputh, Evolutionary Multi-Objective Approach for Resource Allocation in OFDM Systems, $4^{\text {th }}$ International Joint Conference on Computational Sciences and Optimization Kunming, Yunnan China, 15 - 19 April 2011.

[10] K.Deb et al., A fast elitist non-dominated sorting genetic algorithm for multi-objective optimization: NSGA II, Parallel Solving from Nature VI Conference, 16 - 20 September 2000, Paris, France, 849 - 858.

[11] T. Ozel, Intelligent Machining, pp 246 - 265, Wiley, 2009.

[12] S.Gheitanchi et al., Particle Swarm Optimization for Resource Allocation in OFDMA, Proc. International Conference on digital Signal Processing, 2007.

[13] C.K.Chakravarthy and P.Reddy, Particle Swarm Optimization Based Approach for resource Allocation and Scheduling in OFDMA Systems, International Journal of Communications, Network and System Sciences, P 466 -471, March 2010.

[14] D.S.Shu'aibu et al., Dynamic Resource allocation in mobile WiMax using Particle Swarm Optimization Techniques, International Journal of Physical Sciences Vol. 6, pp 1009 - 1014, March 2011.

[15] Yang Yi et al., Modified Particle Swarm Optimization and Genetic Algorithm Based Adaptive Resources Algorithm for Multiuser Orthogonal Frequency Division Multiplexing System, information technology Journal, Asian Network for Scientific Information, March 2011.

[16] I.Ahmed et al., Margin Adaptive resource Allocation for Multi-user OFDM Systems by Particle Swarm Optimization and Differential Evolution, International Journal of engineering \& Technology, February 2011.

[17] S. T. Chung and A. Goldsmith, "Degree of Freedom in Adaptive Modulation: A Unified View." IEEE Trans. Commun., Vol 49 no.9 pp 1561 - 1571, Sept 2001.

[18] J.Kennedy and R.C.Eberhart, Particle Swarm Optimization, Proceedings of IEEE Conference on Neural Networks,Pages 1942-1948,1995.

[19] C. Coello et al., Handling Multiple objectives with Particle Swarm Optimization, IEEE transaction Evolutionary Computation, Vol. 8, No. 3, June 2004.

[20] K.Miettinen, Nonlinear Multiobjective Optimization, 1999, Boston, Kluwer Academic Publishers.

[21] Deb.K, An efficient constraint handling method for genetic algorithm, Computer Methodd in Applied Mechanics and Engineering, p 311-338, 2000.

[22] D. Van Veldhuizen, Multobjective evolutionary algorithms: Classification, analyses and new innovations, Air Force Inst. Technology, Dayton, OH Tech Rep. AFIT/DS/ENG/99-01, 1999

[23] Mehlfuhrer et al. EURASIP Journal on Advances in Signal Processing 2011, 2011-29.

[24] http://www.nt.tuwien.c.at/testsimulator/ 
International Journal of Computer Networks \& Communications (IJCNC) Vol.4, No.4, July 2012

\section{AUTHORS}

Professor HARRY.C.S RUGHOOPUTH (PhD) currently professor at Faculty of Engineering of the University of Mauritius and actively involved in research work.

\section{RAJENDRASINGH ANNAUTH}

Graduated in Electrical and Electronics Engineering (BTECH) and Master of Philosophy (Communications Engineering) from University of Mauritius, he is pursuing research work for $\mathrm{PhD}$. His research interest is optimization of communication resources in OFDM systems using artificial intelligence. He is presently the Divisional Head of the Communication Navigation and Surveillance of the Civil Aviation Department of the Republic of Mauritius

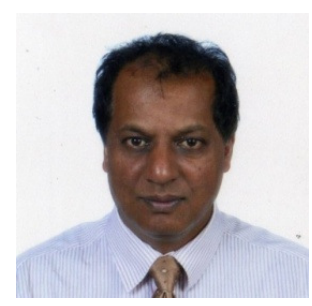

